\title{
This tangled web of reproductive morbidity risk: Abortion stigma, safety \& legality
}

\author{
Bayla Ostrach* \\ Department of Family Medicine, Boston University School of Medicine, Massachusetts, USA
}

\begin{abstract}
The particular combination of political-economic, legal, and sociocultural circumstances in which women seek abortion care has a significant impact on legality, and thus safety. One of the strongest determinants of abortion legality, and in turn, safety, is the degree of stigma attached to it. As such, abortion stigma should be considered, acknowledged, and addressed as a predictive factor in abortion safety and in reproductive morbidity and mortality risk. The purpose of this literature review and analysis was to identify global factors that affect the likelihood of women in a given setting being able to access safe abortion, with an eye to reducing the globally recognized public health risks of negative outcomes from illegal, clandestine, and/or unsafe abortion. Improving access to safe abortion services and reducing morbidity and mortality related to unsafe abortion is critical to public health worldwide. Women, particularly from marginalized populations, have more access to safe abortion where the procedure is: 1) subject to fewer legal restrictions, 2) less stigmatized, and 3) covered by a public health system that offers at least some level of health care to all or most residents. Addressing the various factors that interfere with women's ability to access safe abortion and those that stem from and contribute to abortion-related stigma in particular, is necessary to reduce reproductive morbidity and mortality and improve reproductive health internationally. To successfully advocate for improvements in women's health globally demands a greater understanding of the multi-faceted causes and predictors of abortion stigma, abortion illegality, and the related increased likelihood of unsafe abortion.
\end{abstract}

\section{Introduction}

People across the globe seek abortion in a diverse range of settings. The quality and accessibilityof care is determined by the diverse range of circumstances in which it is sought, which are shaped by larger sociopolitical, economic, and cultural structures affecting those who seek it. Abortion may be legal or illegal, restricted or quite liberal, safe or less so, provided free of charge in a national health system or available only clandestinely, self-induced, socially sanctioned and stigmatized or widely acceptable, publicly funded and covered by insurance, or very expensive [1-4]. This particular combination of political-economic, legal, and sociocultural circumstances in which one seeks care has a significant impact on the ability to obtain a safe abortion. Ensuring access to high quality, safe procedures has the potential to reduce the globally recognized public health burden of negative outcomes from unsafe abortion [5]. The purpose of the literature review and analysis described here was to identify and explore relationships between the broad set of cross-cultural factors that most dramatically affect the likelihood of being able to access safe abortion.

Obtaining an abortion may entail navigating legal, socioeconomic, and other obstacles to care $[6,4,7]$. Improving access to safe abortion services is critical for reducing reproductive morbidity and mortality in the form death or injury from pregnancy and abortion or postabortion complications. Cross-culturally, women, particularly those from marginalized populations such as immigrants, rural women ${ }^{1}$, those with less access to providers, teens, and those most affected

${ }^{1}$ Out of regard for current discussions of gender inclusivity and reproductive justice, I wish to acknowledge that transmen and others besides ciswomen also seek abortion care. However because the majority of available literature on abortion stigma, safety, and legality discusses reproductive morbidity and mortality risks for "women" throughout most of this article I refer to those seeking abortion and experiencing such risks as women. by structural violence, ${ }^{2}$ have more access to safe abortion where the procedure is:

\section{1. subject to fewer legal restrictions}

\section{2. less stigmatized, and}

3. especially where it is covered by a public health system that offers at least some level of health care to all or most residents of a region, without charging for services.

Successfully improving reproductive health internationally, across cultural settings, will thus require all of the following:

- Identifying and addressing factors that interfere with optimal access to safe, high quality abortion procedures, performed by properly trained providers in clean, sterile settings with appropriate sterilization of instruments and/or safe medications with proper follow-up care available;

- Recognizing and addressing bidirectional factors in abortion access that stem from and contribute to abortion-related stigma [8] in particular;

${ }^{2}$ Defined by Farmer, Connors, and Simmons [2008:341] as, "a series of largescale forces - ranging from gender inequality and racism to poverty - which structure unequal access to goods and services," including health care.

Correspondence to: Bayla Ostrach, MA, PhD, Department of Family Medicine, Boston University School of Medicine, Massachusetts, USA, E-mail: ostrachb@bu.edu

Key words: Stigma, legal abortion, abortion, unsafe abortion, morbidity, mortality, pregnancy

Received: March 16, 2016; Accepted: April 11, 2016; Published: April 14, 2016 
- $\quad$ Recognizing and addressing interacting and overlapping relationships between any of these factors that increase the overall disease burden of resulting complications; and

- Addressing they ways that all of these factors and their interactions deleteriously affect global health, in order to reduce overall reproductive morbidity and mortality.

Complications from unsafe and/or illegal abortion are responsible for $13 \%$ of 'maternal' [pregnancy-related] deaths annually [3]. Further underscoring the magnitude of the staggering need for greater access to safe abortion, an estimated 215 million women in the developing world wish to prevent pregnancy but have an "unmet need for modern contraceptives" [9:1]. To successfully improve reproductive health globally thus demands a greater understanding of the multi-faceted causes, consequences, and predictors of abortion stigma - and the related increased likelihood of unsafe abortion or reduced access to safe abortion.

Throughout this discussion, I use the term "abortion stigma" to refer to Kumar et al.'s 2009 description,

Abortion stigma has been defined as "a negative attribute ascribed to women who seek to terminate a pregnancy that marks them, internally or externally, as inferior to ideals of womanhood

as cited in a more recent publication [8:1], where the author powerfully reminds us to consider abortion-related stigma not as a catch-all term for any negative emotion experienced or perceived in relation to difficulty obtaining an abortion, but rather to identify and closely examine precisely the structural factors that interact bidirectionally with both stigma and discrimination to shape access and experience. That is therefore the task at hand in this article.

As I will demonstrate in the following sections, there does exist literature showing that those exposed to abortion-related stigma; those discredited and treated differently for seeking and/or obtaining an abortion, and/or denied the access to safe abortion that may be available to those less or not affected by abortion stigma, are more likely to suffer physical, economic, and emotional effects of forced childbearing, and the morbidity and mortality resulting from complications associated with unsafe and/or legally restricted abortion.

\section{Abortion need worldwide}

Half of all pregnancies worldwide are unplanned [9] and at least $20 \%$ of all pregnancies end in abortion [10]. Despite the consistent nature of such statistics, recent studies confirm that abortion rates are somewhat higher in countries where the procedure is illegal or heavily restricted [11]. About half of abortions performed worldwide are unsafe $^{3}$ - procedures performed in less than optimal conditions pose serious health risks including infection, chronic pelvic pain, infertility, and death for women worldwide [11,3,10].

Applying a medical-anthropological perspective, rather than solely epidemiological or clinical approaches, to an examination of factors in abortion safety allows for a fuller illumination of people's lived experiences with the various economic, logistical, and social obstacles

${ }^{3}$ That said, not all illegal abortions are unsafe, and not all unsafe abortions are illegal [5]- the important determinant factor is the training of the providers and the quality of the care provided, and so it is important even in the context of a literature to be specific in the terminology. Illegal and/or unsafe abortion refers to any abortion provided in less than optimal clinical settings, which may be a legal abortion provided in a less than sterile environment, or by a less-skilled provider, or it could be a high-quality abortion provided somewhere that the procedure is heavily restricted. to abortion access that increase risks for reproductive morbidity. Such attention to cross-cultural contexts and multidimensional realities can help inform approaches to expand access to safe, accessible, reproductive health care overall. One of the strongest patterns to emerge through my review and analysisis that of the compelling inter-relationships between abortion-related stigma, abortion legality, and abortion safety, as Kumar suggests [8]. In particular, there is an apparent bidirectional, interacting, role between abortion stigma and illegality in producing or increasing risks for reproductive morbidity. Particular consideration of abortion-related stigma as a key factor influencing access to both safe and legal abortion is critical for taking steps to improve abortion access overall. To safeguard women's health, in order to reduce the global burden of reproductive morbidity, we must begin to untangle this tightly gnarled web of abortion stigma, legal restrictions, lack of safety, and abortion complications.

\section{Methods}

Through keyword searches of several widely used online databases of medical and anthropological peer-reviewed literature performed sequentially over an initial six-month period ${ }^{4}$, I originally reviewed relevant literature to identify various factors that appeared to significantly affect women's access to safe abortion internationally, including: legality, stigma, safety, availability, and cultural/religious constraints. Through an iterative, inductive process, I then re-reviewed all relevant literature found through these searches to more closely examine relationships between the considered factors in order to develop a conceptual framework [12] for evaluating the roles of various factors in international abortion access. The resulting framework proposed here reveals a range of socio-cultural and political-economic factors that shape abortion access across regions. The relationships between factors I propose here is not intended to be exhaustive or to address all possible variations, but rather reflects the literature identified and reviewed through the methods described, and my medical-anthropological analysis of the implications of the identified, intersecting factors as they bear out in people's lived experiences with abortion stigma and reproductive morbidity risk.

\section{Conceptual framework}

This review and analysis of existing relevant literature at the time I evaluated it revealed a close connection between abortion-related stigma, abortion [il]legality, and abortion safety -- worldwide and in varying cultural contexts. Research and commentaries published since the initial review $[5,13,14]$ further illustrate that it is not as simple as legal $=$ safe or illegal $=$ unsafe in the world of abortion care. Rather, legal abortion can in some settings be of low quality (particularly where heavily stigmatized or restricted), and illegal abortion can be safe, when it is high quality care, offered with good support by trained, qualified providers (e.g. Women on Waves, Women on Web). That said, at the time the literatures discussed here were reviewed the a strong relationship between stigma, legality, and safety did emerge and thus this relationship is explored and presented at length and in detail.

One of the most important patterns to emerge was in fact a [re] confirmation of prevailing wisdom among providers and researchers in the field of abortion care that abortion legality is a strong predictor of abortion safety, as well as of abortion accessibility $[4,15,10,16]$. Both legality and safety have been previously associated with the level of abortion-related stigma $[17,18]$, though the impacts of these

${ }^{4}$ The literature review and analysis described here was originally undertaken as part of a comprehensive doctoral examination. It has since been periodically updated for the purposes of manuscript preparation. 
connections on resulting morbidity and mortality have not been comprehensively explored. Reproductive morbidity resulting from unsafe abortion is not only a direct sequelae of non-sterile or untrained abortion methods, but can also be the result of abortion illegality, as having limited or no access to legal abortion may increases women's likelihood of receiving unsafe or clandestine abortion services, with accompanying risks [14]. Women exposed to abortion stigma and denied safe abortion are more likely to suffer physical, economic, and emotional effects of forced childbearing; or to suffer morbidity and mortality resulting from complications associated with clandestine, unsafe, and/or illegal abortion [13].

Researchers estimate that up to $40 \%$ of the world's female population lives in countries where abortion is illegal, though the African continent, Central and South America, and some parts of Asia are likely over-represented in this estimate [19]. This exposes the subset of women of reproductive age in these populations to increased risks of mortality and morbidity related to unsafe abortion and abortion stigma. The legality of abortion in different geographic regions of the world also varies widely, with a greater frequency of legal abortion noted in European, North American, and Asian countries.

At the time of the initial literature review upon which this conceptual framework is based, abortion was illegal or heavily restricted in most African countries [20,7,21-23], with the exceptions of South Africa [24], Tunisia [25], and possibly soon, Sierra Leone [26]. In the case of Sierra Leone, the Parliament voted overwhelmingly in December 2015 to make abortion legal in the first trimester of pregnancy for the first time in that country, but the president refused to approve the new law, and sent it back to Parliament, where it now remains in limbo [26].

In Latin America and the Caribbean, abortion is illegal or restricted in most countries [18,19,26-28]. Notable exceptions are some parts of Mexico including the Distrito Federal [29], Colombia [30], Uruguay under limited circumstances [31], Puerto Rico and a few other Caribbean islands [32], including Cuba [33].

In Europe, abortion is legal and widely available at least in the first trimester in many countries, but not all [17,29,30,34-36]. Spain, and autonomous nations considered part of Spain [e.g. Catalunya] joined most of their European Union neighbors when 2010 reforms made abortion for any reason in the first trimester legal, with some limitations maintained after that point [32], though this new ruling was immediately threatened to be overturned at any time by the subsequently elected right-wing conservative government in Madrid, which repeatedly vowed to reverse reforms and in fact restricted legal access for minors in late 2015 [37]. While many women in Europe can access early legal abortion, often through national or public health systems, women in need of second-trimester care face more obstacles and delays [38].

\section{Global patterns in abortion-related stigma, legality, and safety}

Data on abortion-related stigma are unavailable for many countries, but certain patterns are apparent. A comparatively higher level of abortion-related stigma is documented in Africa, where abortion is heavily restricted or illegal, and frequently unsafe, with few exceptions. However, in Tunisia, a rare example of an African country where abortion is legal, abortion stigma is relatively lower [25]. Perhaps this rare example of legal abortion in this African country persists because levels of stigma are comparatively lower, or perhaps stigma is lower because the procedure is still legal. The bidirectional relationship between lower levels of abortion stigma and greater abortion legality, where these overlap, warrants further study.

\section{North America}

Abortion in North America tends to be very safe where it is legal, with estimated complication rates at less than $0.01 \%$ for early procedures in the U.S. [1]. Nonetheless, high levels of abortion-related stigma may deter women from seeking abortion follow-up care when needed, even where it is legal [17]. Women delayed into the second trimester of pregnancy because of various obstacles [including a reported fear of encountering negative reactions from partners, family, and protesters outside clinics - tangible evidence of anti-abortion stigma] face increased risks of complications the later into a pregnancy they are delayed $[32,35]$.

Abortion stigma is relatively low in Canada (compared to the Unites States), where abortion is legal and offered through a national health system [39,40]; Johnson et al. 2004. In the United States, where abortion restrictions vary by state but are present in almost all, and where women encounter many obstacles to care despite the legal and constitutional right to seek it [1] abortion stigma serves as a persistent barrier to care or source of delays [16].

\section{Asia}

In Asia, abortion-related stigma is lower in countries where abortion is legal, and variations in both legality and stigma appear to more clearly correlate with religious attitudes, based on the available literature [22,41]. In Asian countries where abortion is heavily restricted and/or widely unavailable, levels of abortion-related stigma appear to be high $[42,43]$. While India's regional public health systems cover abortion, many women reportedly seek abortion primarily from private, often clandestine, providers, in part because in rural areas it may be harder to reach legal providers and because of a lack of widespread information about the option of legal abortion [41]. In South India, where public health programs are widely used, abortion is not as stigmatized [43] perhaps due also to the relatively greater gender equality in states like Kerala. Bangladesh is a curious case, as abortion is illegal in almost all circumstances - yet women are able to access relatively safe early abortion by seeking a similar procedure, referring to it as 'menstrual extraction' [42]. The presence of an alternate abortion method seemingly accepted as an approach to 'miscarriage management' or used to bring on a late period offers a less-stigmatized avenue for women seeking safe abortion, even in the context of abortion illegality [42]. This is one example of the illegal-does-not-always-meanunsafe conundrum to which Ganatra, Gerdts, and others refer.

Abortion is safe or relatively safe in Vietnam, where it is covered by the national health system [21]. In Nepal, abortion is relatively safe for women who can afford to go to private clinics for a legal procedure, but $20 \%$ of "maternal" (i.e. pregnancy-related) deaths there are still attributed to complications from clandestine care sought by Nepalese women who cannot afford private care, or who continued to assume abortion was illegal after it was legalized [41].

Abortion stigma can be so powerful that women assume a legal procedure is unavailable, and seek it through extra-legal, potentially lower quality, less-safe avenues. That said, Asian countries with legal abortion and low levels of abortion stigma, or where abortion is available under the guise of a less-stigmatized procedure such as menstrual extraction, also tend to have very low abortion complication rates. 


\section{Latin America and the Caribbean}

In Latin America, widespread use of folk remedies and herbal preparations, or off-label use of medications obtained over the counter [all used to self-induce an abortion] may contribute to under-reporting of abortion complications and higher rates of unsafe abortion [26,19]. Even in the few places in Latin America where abortion is legal, unsafe abortion is a serious public health concern and safe abortion in a clinical setting is often difficult to obtain $[13,17,24,27,44,45]$.

Abortion is legal and less-stigmatized in Cuba and Puerto Rico, two of the few places in Latin America and the Caribbean where the procedure is legal and available, while even in other Latin American and Caribbean countries where abortion is decriminalized or legal in some areas or under certain conditions, stigma is still a significant obstacle to safe abortion $[13,17,27,24,44]$.

\section{Europe}

In Europe, abortion stigma is relatively low in the many countries where abortion is legal and offered through public health systems at least in the first trimester, as compared to countries where it is illegal or restricted (e.g. [34]). There is regional variability, with some Eastern European countries experiencing higher rates of abortion stigma, for example Poland [40]. Nevertheless, abortion is quite safe in much of Europe, due in part to the relative lack of legal restrictions at least in the first trimester, and the availability of public health systems in most countries that typically include at least some level of coverage for early abortion [28,46,37].

Spain and the autonomous regions considered part of Spain (e.g. Catalunya) joined most of their European Union neighbors in 2010 when abortion for any reason became legal in the first trimester, with specific limitations maintained after that point. However, this new ruling was repeatedly threatened with being overturned by the subsequent conservative government in Madrid [33].

As a notable exception to the relatively widespread legality of [at least first-trimester] abortion in Europe, levels of abortion-related stigma are high in Ireland, where abortion is entirely prohibited and culturally taboo, in the context of conservative Catholicism [41]. Thousands of Irish women and immigrant women in Ireland seeking abortion travel to England each year for the procedure, producing an additional economic burden related to travel costs and time away from work [47]. Abortion is also more stigmatized and less-accessible in Poland, where women must pay privately for the procedure, meaning access is stratified by class [45]. Abortion is illegal under all or most circumstances (only to save the life of the woman) in Ireland, Andorra, Malta, Monaco, and San Marino [48] though some women from these countries travel to other European nations to seek abortion (author's unpublished fieldnotes).

\section{Africa}

Africa has unfortunately been largely left out of this part of the discussion, as few countries in the region offer legal abortion, and there was a comparable dearth of information about abortion accessibility on the African continent at the time the literature review was conducted though no less global emphasis on the impact of reduced access to safe abortion on health and safety. The African Charter on the Rights of Women [43] enjoins African countries to take appropriate measures to protect the reproductive rights of women by allowing abortion in cases of sexual assault, rape, incest, and when a pregnancy endangers the health or life of the woman or her fetus, yet based on available literature at the time of the review described, Tunisia and South Africa appeared to be the only African countries where abortion was both legal and accessible [5,13]. Sierra Leone is now also on the brink of abortion legalization, pending the current president's (unlikely) approval of a popular late 2015 parliamentary bill [25]. With such reduced access to legal and/or safe abortion, the continent of Africa still accounts for half of all deaths related to unsafe abortion worldwide [9].

Overall, it appears that in terms of legal restrictions, where women must obtain government or provider approval for exceptions to prohibitions on abortion, such as in cases of rape, incest, and other 'extenuating' circumstances, these requirements often result in delays or denials of care. Such delays and outright prohibition can compromise confidentiality and result in increased risks for complications, while legal restrictions on abortion generally compromise abortion safety, worldwide.

\section{Contexts of abortion stigma: Culture, politics, \&gender roles}

Various cultural and political settings or factors can increase the risks for or impacts of abortion-related stigma, resulting in greater negative mental and physical health effects for women who encounter stigma, and whose access to safe abortion is thus reduced $[15,16]$. 'Culture' as a factor in access to various forms of health care is poorly represented in epidemiology. This is an area where contributions from medical anthropology and other ethnographic disciplines are sorely needed, and of great value.

In any setting, the impact of particular cultural dynamics, including the role of religious traditions, may affect abortion legality and safety, abortion-related stigma, and relationships between these. An analysis of the limited literature on cultural factors reveals locally specific but also globally recurring patterns in abortion access. Abortion-related stigma is closely linked to cultural attitudes about gender roles for women, especially with regards to pregnancy, in settings where culture and religion shape gender role expectations [16]. Across most societies, cultural beliefs and values surrounding women's identities as mothers and nurturers appear to impact the degree to which women are trusted with decision-making about unplanned or ill-timed pregnancies, versus ingrained cultural or societal expectations that husbands or fathers, or even (predominantly) male doctors have the ultimate say in determining the course of a pregnancy (e.g., Kumar et al., 2009).

In more patriarchal countries and cultural settings where the number of children a woman has is important to her husband's status, as is often assumed to be the case in much of the Middle East and Asia, and/or where cross-cultural ideas about women's 'natural' or expected role as mothers predominate and are reinforced by social policy that does not adequately compensate domestic labor, such cultural values and attitudes may be assumed to serve as prohibitive barriers to access for women who would seek to terminate an illtimed pregnancy, or may, conversely, motivate women to secretly seek abortion even when doing so is medically and/or socially risky or involves pursuing clandestine methods. An awareness that cultural and religious attitudes about abortion serve as powerful sources of stigma in places where abortion is illegal, restricted, or difficult to obtain can also inform an understanding of the lengths that women will go to in order to obtain clandestine abortion despite associated risks for reproductive morbidity. These are areas ripe for further ethnographic and anthropological research, with an ever-present awareness of the great sensitivity to cross-cultural variations in belief systems, and emic perspectives needed. 


\section{Political and cultural constraints}

Structural violence is a term often heard in medical anthropology and originally used by Johann Galtung in 1969 to describe the ways that institutionalized gender, class, and ethnic inequalities are maintained and perpetuated by governments and institutions, such as health systems, education, and social service bureaucracies and those in power, to negatively impact people's lives [20]. Structural violence is recognized as an important driver of various political-economic factors in abortion access [1,7] and shapes cultural and social attitudes and expectations related to pregnancy-related behavior. Moreover, political forces and culturally shaped structural constraints that compel the continuation of an unwanted pregnancy and force women to raise a child they do not feel prepared to care for when they cannot access abortion must be seen as a further form of structural violence that affects physical and mental health. Women are already more likely than men to live in poverty internationally, so each setback or challenge that disproportionately increases women's likelihood of ending up in, or deeper in, poverty [such as a unique biological vulnerability to pregnancy] exacerbates this dynamic. Additionally, the experience of single motherhood or parenting in the absence of adequate social support, can keep or push women and their children into socially marginalized categories where they are more affected by various forms of institutional oppression and deprivation than are their biologically male counterparts.

For example, preventing or delaying women in poverty in the United States from obtaining federally-entitled Medicaid coverage that [in some states], pays for abortion is a specific form of structural violence that effectively deprives people already affected by gender inequality and economic deprivation of access to legal abortion and other forms of health care [4,1] [Ostrach \& Cheyney, 2014]. By affecting people across a broad spectrum of settings where abortion legality and availability vary widely, abortion-related stigma plays a notably strong role in increasing the risks of morbidity related to abortion, often regardless of the particular legal restrictions on abortion, or lack there of, in a given setting. When even a woman who ostensibly has full legal access to abortion cannot in fact obtain an abortion, merely because she is more likely to be in poverty due to her gendered social status and lower earning potential, and then has difficulty obtaining Medicaid coverage for an abortion due to the abortion stigma that uniquely affects pregnant women in need of Medicaid coverage for abortion services, this doubly gendered form of discrimination exemplifies multiple ways that structural violence impacts the role of abortion stigma in influencing abortion legality, accessibility, safety, and access.

\section{Deleterious health effects of abortion stigma}

Abortion-related stigma, and the ways it is enacted upon those who seek or obtain abortion [49,50], is a particularly insidious and destructive form of structural violence insofar as it threatens physical and emotional health, and status, and affects people already disproportionately vulnerable to physical and social suffering on biological, economic, political, social, and medical levels [51]. Anything that further predisposes women to greater risks for being forced to carry an unwanted pregnancy to term, or to suffering physical and mental health complications from unsafe abortion and abortion stigma, exacerbates such vulnerabilities.

The vast majority of abortion-related deaths and serious injuries resulting from illegal and/or unsafe abortion occur in the developing world - while illegal abortion is not always unsafe, and vice versa, seeking abortion under circumstances of illegality disproportionately means women risk grave injury or death resulting from unsafe abortion $[9,19]$. When people do not have straightforward, unstigmatized access to legal, openly available, safe, high quality abortion services, they may feel compelled to seek lower quality, clandestine, often unsafe care [52,53].

Carrying an unplanned or ill-timed pregnancy to term can also result in poor mental or physical health, or psychosocial outcomes -- in one recent study, teen women who carried an unplanned pregnancy to term had higher rates of depression, low self-esteem, and low selfworth than their counterparts who obtained abortion [54-61]. Ongoing long-term research evaluating the physical, economic, financial, and psychological impacts of being denied a wanted abortion on women and the children they are forced to bear identified a greater likelihood that such women are statistically more likely to be in poverty, raising (a) child(ren) without partner or family support, and/or more likely to be in an abusive relationship, several years after being denied an abortion, among other troubling health and social consequences of being denied a wanted abortion [62].

Together, these findings suggest that the negative effects on mental and physical health that result from abortion-related stigma must be seen and measured as particular forms of reproductive morbidity that uniquely impact women affected by abortion stigma. By increasing risks for negative mental and physical health sequelae related to stigmatized, unsafe, and/or illegal abortion, abortion stigma makes some abortions less safe, or makes abortion more likely to be illegal, in some settings. When the immediate negative health impacts of abortion-related stigma are considered alongside the negative physical health effects and reproductive morbidity risks of unsafe abortion, the overall health burden of abortion stigma as it affects abortion safety [often as a function of illegality] is clear. ${ }^{5}$

\section{Discussion}

The strong bidirectional relationship between abortion stigma and abortion legality is evident in the literature discussed above. High levels of stigma are strongly correlated with abortion being illegal, while illegal abortion appears to be more highly stigmatized. Illegal abortion also tends to be less safe. A growing body of research increasingly documents how the emotional and psychological effects of abortion-related stigma, though not abortion itself, have significant impacts on mental and physical health [16,55]; and on perceptions of the availability of safe abortion [24]. When women perceive high levels of abortion stigma, they may perceive abortion as less accessible, or assume it is illegal even when it is not [24]. I also suggest that when women are more affected by abortion stigma while obtaining care, they may be less likely to comply with follow-up instructions or to seek needed attention for rare but serious complications, increasing risks for various preventable but harmful sequelae [see Ostrach \& AbiSamra, forthcoming].

The literature reviewed for this project, evaluated together, overwhelmingly demonstrate that abortion-related stigma is a key factor in the persistent illegality and un-safety of abortion in many regions of the world, especially in Asia, Africa, Latin America, and some parts of Europe -- despite global recognition of links between abortion legality and safety. Even though the World Health Organization and other global bodies widely acknowledged as leading experts on

${ }^{5}$ The particular interactions between structural abortion-related stigma, difficulty complying with follow-up care instructions or seeking aftercare, and resulting complications such as infection or hemorrhage, producing a stigma-related reproductive health syndemic, are the focus of ongoing research by this author and a colleague, forthcoming elsewhere. 
reproductive safety and health policy have identified reducing illegal and unsafe abortion, and increasing access to legal, safe abortion as global priorities, nations strongly affected by abortion stigma continue to maintain and promote abortion restrictions - risking the health of their residents. Much new literature has been published since the initial review conducted for this project, which appears to only more strongly support the arguments advanced here.

The persistent power of abortion stigma, as it is used to justify making abortion less safe and less legal in order to restrict women's health and liberty, also lies in the powerful cultural symbolism of certain idealized social characteristics, widely accepted as inherent to women, which abortion "transgresses," [17:4] by potentially allowing women a way to opt out of fulfilling asocially and culturally prescribed maternal role [though of course, most women seeking abortion already have children]. The cultural and gender role expectations upset by such 'transgressions' are, namely, dominant cross-cultural perceptions of women as ideally fertile, maternal, and nurturing under all circumstances. For example, even women in the western United States interviewed about their experiences accessing safe, legal, publicly funded abortion described feeling pressure to overcome negative stereotypes about the 'type' of woman who has an abortion, before feeling able to go to an abortion clinic [1].

The connections between abortion-related stigma and abortion restrictions, and/or between a lack of safe abortion services and the deleterious mental and physical health effects of abortion-related stigma even where the procedure is legal and nominally available illustrate the importance of evaluating the damaging emotional and physical health effects of abortion-related stigma as a particular form of reproductive morbidity risk that results from gender role expectations and structural violence disproportionately affecting women worldwide. The threat of this sociocultural and political-economic form of reproductive morbidity risk influences overall health risks, contributing to global reproductive morbidity and mortality burdens.

Gendered power dynamics at the family, community, societal, and cultural levels mean that decision-making about pregnancies is also constrained by cross-culturally varying ideas about who in a family or marriage makes decisions about family planning [57] or by the availability of traditional methods that may be considered or not, in part based on transmission of knowledge shared inter-generationally by women. In any country or setting where abortion is stigmatized, restricted, unsafe, or illegal, women may risk their health or their lives to obtain clandestine abortion services in spite of, or perhaps because of, the varying cultural and religious pressures that limit access to safe abortion.

In settings where abortion is highly stigmatized, healthcare providers' attitudes about abortion [informed and shaped by their own cultural expectations] may also influence access to care [58]. Recent research [59] further confirms the deleterious effects of abortion-related stigma on women's health and safety, concluding that stigmatization of abortion may in fact limit the number of providers trained and prepared to provide safe abortion and abortion follow-up services, endangering those worldwide who seek care but cannot find a skilled provider. Equally troubling, abortion-related stigma may deter some from obtaining a wanted abortion, instead forcing them to carry an unwanted pregnancy to term, which in turn increases women and children's risks of ending up in poverty, abuse, or other vulnerable situations [54].
When we understand abortion-related stigma as not only a social or cultural phenomenon, but as something deliberately or unconsciously structured and institutionalized through structural violence and cultural conditioning as part of gender role expectations, the power of such stigma to serve as a barrier to safe abortion access and as a threat to health and safety becomes impossible to ignore. The impact of stigma on access to safe abortion persists cross-culturally and globally. In countries where abortion is illegal and/or unsafe, structural violence serves as a useful framework for defining the subjugated political and social statuses of those who are often denied the ability to make decisions about their own health and childbearing plans, as well as describing larger political-economic contexts that unjustly keep women, particularly 'third-world' and women of color, in poverty; more likely to encounter obstacles to safe abortion $[1,21,60]$.

Of course, some of these factors are also at play even in settings where abortion is nominally legal but complicated to access, particularly when high levels of stigma are noted, such as in the United States $[16,50,61]$.

Structural violence has the potential to affect reproductive morbidity when poverty, low social status, and institutionalized sexism [as seen in gender pay gaps, greater vulnerability to violence, and greater likelihood of being in poverty], prevent women from accessing legal and safe abortion, thereby increasing risks for abortion-related morbidity and mortality caused by delayed or unsafe abortions. Even where abortion is legal and can be accessed safely, the most socially and economically marginalized women are more likely to encounter delays and obstacles that may relate to various forms of stigma $[1,16,17,50,61,62]$.

\section{Conclusion}

A range of socio-cultural and political-economic factors, not least of these being stigma, structure access to safe, high-quality abortion. All of these interlocking and mutually reinforcing elements carry significant health implications, resulting in a tangled web of stigma, lack of safety, restrictions, and risk. When legal restrictions and abortion-related stigma present obstacles to abortion access, affect abortion safety, and increase risks for suffering from complications related to unsafe abortion, the economic, political, social, and cultural contexts that produce such obstacles are responsible for negative health outcomes in the form of increased reproductive morbidity.

Institutionalized gender role expectations, including cultural norms that dictate women's 'ideal' roles as mothers, and the widespread poverty that disproportionately affects women, function as forms of structural violence that produce and promote abortion-related stigma, maintain or contribute to conditions of abortion illegality and/or unsafety, and constrain women's options for making decisions about the course of their reproductive lives. For women in poverty, living under conditions of abortion illegality, unavailability, where abortion is unsafe, and/or living in settings where levels of abortion stigma are high, these political-economic and socio-cultural realities limit their lives and choices, negatively affect their health,and increase their risks of dying or being seriously injured by an unsafe abortion. The mental and physical health effects of abortion-related stigma, in addition to the negative physical and social outcomes suffered by women denied abortion [56] must also be taken into account when tallying up the numbers of women at highest risk for reproductive morbidity and mortality and associated suffering.

By this measure, if we consider the physical and emotional 
sequelae of abortion stigma as a form of reproductive morbidity, women in countries where abortion is legal but heavily restricted and/ or stigmatized, such as the U.S., may be just as much at risk, despite a typical stereotyped image of women in developing countries as being more in danger of the deleterious effects of restricted access to safe abortion. Medical literature and research already address the clinical aspects of illegal and unsafe abortion - this article contributes to and calls for a broader perspective on the cultural and social factors that affect abortion-related stigma, and with it, true access to safe abortion and reductions in reproductive morbidity and mortality.

The conceptual framework developed through this review and analysis reveals the wide range of socio-cultural and political-economic factors that shape abortion access, internationally. This framework specifically demonstrates that an increased likelihood of reproductive morbidity related to illegal and/or unsafe abortion can be predicted in the followings situations: where legal restrictions on abortion exist, abortion is less accessible, though no less frequent. In such settings, the risks of complications from abortion are greater, and women are more likely to die from unsafe abortion. Where abortion is illegal or heavily restricted, high levels of abortion stigma are usually seen, and where abortion is stigmatized, it appears more likely to be illegal or restricted. Where cultural and religious attitudes prohibit abortion or make it controversial/contested, levels of abortion stigma are higher, the procedure is more likely to be illegal, and safe abortion procedures are harder to obtain. Finally, abortion-related stigma, as a factor unto itself, increases risks for reproductive morbidity, including the welldocumented negative mental health outcomes that result from abortion stigma itself, rather than from the procedure (e.g. research conducted by Cozzarelli and co-authors and by Jocelyn Warren).

In light of these patterns that predict increased risks for reproductive morbidity related to stigmatized, unsafe, and/or illegal abortion, continuing efforts to improve access to safe abortion and reduce deleterious reproductive health outcomes worldwide must acknowledge and address abortion-related stigma, structural violence, and cultural and social factors that limit reproductive health care options and negatively impact reproductive health. It will be impossible to untangle the threads that tie abortion stigma to legality, legality to safety, and each of these to morbidity, in order to meet global reproductive health goals, until we do so.

\section{Authorship}

Bayla Ostrach, MA, PhD is a member of the faculty with the Master's program in Medical Anthropology \& Cross-Cultural Practice at the Boston University School of Medicine, and Assistant Professor in the Department of Family Medicine. A Fellow of the Society of Family Planning, Dr. Ostrach has worked directly in the field of reproductive health and abortion care since 1999. With her colleague Roula AbiSamra, MPH, she has a forthcoming book chapter on abortion stigma syndemics.

\section{Acknowledgements}

This article appears in much-evolved form from a paper that originally benefitted from the excellent and detailed input of Drs. Merrill Singer, Pamela Erickson, Samuel Martinez, Kim Price-Glynn, all at the University of Connecticut, and Dr. Jocelyn Warren, Oregon State University. I greatly appreciate their early feedback and long-time support of my scholarly work. Thanks also to Dr. Brenda Major, UC Santa Barbara, whose comments following an early presentation of my master's research on women's experiences obtaining abortion in
Oregon shaped my initial thinking about the role of social stigma in abortion accessibility.

\section{Funding}

This literature review and analysis article is based on wholly unfunded research. There are no competing interests.

\section{References}

1. Ostrach B, Cheyney M (2014) Navigating Social and Institutional Obstacles: LowIncome Women Seeking Abortion. Qual Health Res 24: 1006-1017. [Crossref]

2. Ostrach B (2014) Did Policy Change Work? Oregon Women Continue to Encounter Delays in Medicaid Coverage for Abortion. Anthropol Action 21: 20-30.

3. Barot S (2011) Unsafe abortion: the missing link in global efforts to improve maternal health. Guttmacher Policy Rev 14: 24.

4. Boonstra HD (2007) The heart of the matter: public funding of abortion for poor women in the United States. Guttmacher Policy Rev 10: 12-16.

5. Ganatra B, Tunçalp Ö, Johnston HB, Johnson BR Jr, Gülmezoglu AM, et al. (2014) From concept to measurement: operationalizing WHO's definition of unsafe abortion Bull World Health Organ 92: 155.[Crossref]

6. Jones BS, Weitz TA (2009) Legal barriers to second-trimester abortion provision and public health consequences. Am J Public Health 99: 623-630. [Crossref]

7. Ellison MA (2003) Authoritative knowledge and single women's unintentional pregnancies, abortions, adoption, and single motherhood: social stigma and structural violence. Med Anthropol $Q$ 17: 322-347. [Crossref]

8. Kumar A (2013) Everything is not abortion stigma.Womens Health Issues 23: e329331. [Crossref]

9. Darroch JE, Sedgh G, Ball H. Contraceptive technologies: Responding to women's needs. N Y GuttmacherInst [Internet]. 2011 [cited 2015 Sep 10]; Available from: http:// alanguttmacherinstitute.com/pubs/Contraceptive-Technologies.pdf

10. Billings DL, Moreno C, Ramos C, González de León D, Ramírez R, et al. (2002) Constructing access to legal abortion services in Mexico City. Reprod Health Matters 10: 86-94. [Crossref]

11. Sedgh G, Singh S, Shah IH, Ahman E, Henshaw SK, et al. (2012) Induced abortion: incidence and trends worldwide from 1995 to 2008. Lancet 379: 625-632. [Crossref]

12. Bryant A, Charmaz K (2010) The SAGE Handbook of Grounded Theory: Paperback Edition. SAGE.

13. Gerdts C, Vohra D, Ahern J (2013) Measuring unsafe abortion-related mortality: a systematic review of the existing methods. PLoS One 8: e53346. [Crossref]

14. Gerdts C, DePiñeres T, Hajri S, Harries J, Hossain A, et al. (2015) Denial of abortion in legal settings.J Fam Plann Reprod Health Care 41: 161-163. [Crossref]

15. Alan Guttmacher Institute. State-Level Assault on Abortion Rights Continues in First Half of 2013 [Internet]. 2013 [cited 2016 Mar 11]. Available from: http://www. guttmacher.org/media/inthenews/2013/07/08/

16. Cozzarelli C, Major B, Karrasch A, Fuegen K (2000) Women's experiences of and reactions to antiabortion picketing. Basic Appl Soc Psychol 22: 265-275.

17. Kumar A, Hessini L, Mitchell EM (2009) Conceptualising abortion stigma. Cult Health Sex 11: 625-639. [Crossref]

18. Grimes DA, Benson J, Singh S, Romero M, Ganatra B, et al. (2006) Unsafe abortion: the preventable pandemic. Lancet 368: 1908-1919. [Crossref]

19. Alan Guttmacher Institute. Facts on Induced Abortion Worldwide: In Brief, Fact Sheet November 2015. [Internet]. 2015 [cited 2016 Mar 11]. Available from: http://www. guttmacher.org/pubs/fb_IAW.pdf

20. Delicia F. Clandestine Abortion in Peru, Facts and Figures. Lima Peru Cent Mujer Peru Flora Tristan. 2002;

21. Farmer P. Pathologies of power: Health, human rights, and the new war on the poor [Internet]. Vol. 4. Univ of California Press; 2004 [cited 2015 Sep 10]. Available from: https://books.google.com/books?hl=en\&lr=\&id=2sbP7J-lckoC\&oi=fnd\&pg=PR11\&d $\mathrm{q}=$ farmer + pathologies + of + power\&ots=YYcsOIDQ_V\&sig=GYPOUcWvQcrfs6lkg 8 GwLu2rfvl

22. Gallo MF, Nghia NC (2007) Real life is different: a qualitative study of why women delay abortion until the second trimester in Vietnam. Soc Sci Med 64: 1812-1822. [Crossref] 
23. Okonofua FE, Odimegwu C, Ajabor H, Daru PH, Johnson A (1999) Assessing the prevalence and determinants of unwanted pregnancy and induced abortion in Nigeria. Stud Fam Plann 30: 67-77. [Crossref]

24. Varkey SJ (2000) Abortion services in South Africa: available yet not accessible to all. Int Fam Plan Perspect 26: 87-88.

25. Hajri S (2004) Medical abortion: the Tunisian experience. Afr J Reprod Health 8: 6369. [Crossref]

26. Ipas. Sierra Leone Parliament votes to reform abortion law. [Internet]. 2015 Dec [cited 2016 Feb 17]. Available from: http://www.ipas.org/en/News/2015/December/SierraLeone-Parliament-votes-to-reform-abortion-law.aspx

27. Singh S, Prada E, Kestler E (2006) Induced abortion and unintended pregnancy in Guatemala. Int Fam Plan Perspect 32: 136-145. [Crossref]

28. Guedes AC (2000) Abortion in Brazil: legislation, reality and options. Reprod Health Matters8: 66-76. [Crossref]

29. Becker D, Díaz Olavarrieta C (2013) Decriminalization of abortion in Mexico City: the effects on women's reproductive rights. Am J Public Health 103: 590-593. [Crossref]

30. Amado ED, CalderónGarcía MC, Cristancho KR, Salas EP, Hauzeur EB (2010) Obstacles and challenges following the partial decriminalisation of abortion in Colombia. Reprod Health Matters 18: 118-126. [Crossref]

31. Singh S, Wulf D,Hussain R, Bankole A, Sedgh G, et al. (2009) Abortion worldwide: a decade of uneven progress. [Internet]. Guttmacher Institute [cited 2015 Sep 10]. Available from: http://www.cabdirect.org/abstracts/20093357525.html

32. Henshaw SK, Singh S, Haas T (1999) The incidence of abortion worldwide. Int Fam Plann Persp 25: S30-38. [Crossref]

33. Veeken H (1995) Cuba: plenty of care, few condoms, no corruption. BMJ 311: 935937. [Crossref]

34. Helström L, Odlind V, Zätterström C, Johansson M, Granath F, et al. (2003) Abortion rate and contraceptive practices in immigrant and native women in Sweden. Scand J Public Health 31: 405-410. [Crossref]

35. Jones EF, Forrest JD, Goldman N, Henshaw SK, Lincoln R, et al. (1985) Teenage pregnancy in developed countries: determinants and policy implications. Fam Plann Perspect 17: 53-63. [Crossref]

36. Johnson BR, Horga M, Fajans P (2004) A strategic assessment of abortion and contraception in Romania. Reprod Health Matters 12: 184-194. [Crossref]

37. Alan Guttmacher Institute. Spain Expands Legal Access to Abortion. [Internet]. 2010 Mar. Available from: www.guttmacher.org/media/inthenews/2010/03/03/index.html

38. Ostrach B (2014) Critical medical anthropology as a roadmap -- Understanding access to abortion in the Catalan health system. Med Anthropol $Q$.

39. Ostrach B (2013) "Yo no sabía..."-immigrant women's use of national health systems for reproductive and abortion care. J Immigr Minor Health 15: 262-272. [Crossref]

40. League CARA (2003) Protecting abortion rights in Canada: a special report to celebrate the 15th anniversary of the decriminalization of abortion. Ott CARAL.

41. Varkey P, Balakrishna PP, Prasad JH, Abraham S, Joseph A (2000) The reality of unsafe abortion in a rural community in South India. Reprod Health Matters 8: 83-91. [Crossref]

42. Shakya G, Kishore S, Bird C, Barak J (2004) Abortion law reform in Nepal: women's right to life and health. Reprod Health Matters 12: 75-84. [Crossref]

43. Ahmed MK, Rahman M, Van Ginneken J (1998) Induced abortions in Matlab, Bangladesh: trends and determinants. Int Fam Plan Perspect: 128-132.
44. Ocran C (2007) Protocol to the African Charter on Human and Peoples' Rights on the Rights of Women in Africa. Afr J Intl Comp L 15:147.

45. Harries J, Orner P, Gabriel M, Mitchell E (2007) Delays in seeking an abortion unti the second trimester: a qualitative study in South Africa. Reprod Health 4: 7. [Crossref]

46. Che ̊,stowskaA (2011) Stigmatisation and commercialisation of abortion services in Poland: turning sin into gold. Reprod Health Matters 19: 98-106. [Crossref]

47. Jackson E, Johnson BR, Gebreselassie H, Kangaude GD, Mhango C (2011) A strategic assessment of unsafe abortion in Malawi. Reprod Health Matters 19: 133-143. [Crossref]

48. Porter E (1996)Culture, community and responsibilities: abortion in Ireland. Sociology 30: 279-298.

49. Boland R, Katzive L (2008) Developments in laws on induced abortion: 1998-2007. Int Fam Plan Perspect 34: 110-120. [Crossref]

50. Cockrill K, Hessini L (2014) Introduction: bringing abortion stigma into focus. Women Health 54: 593-598. [Crossref]

51. Cockrill K, Upadhyay UD, Turan J, Greene Foster D (2013) The Stigma of Having an Abortion: Development of a Scale and Characteristics of Women Experiencing Abortion Stigma. Perspect Sex Reprod Health 45: 79-88.

52. Purcell C (2015) The Sociology of Women's Abortion Experiences: Recent Research and Future Directions. Sociol Compass 9: 585-596.

53. Banerjee SK, Andersen KL, Warvadekar J (2012) Pathways and consequences of unsafe abortion: A comparison among women with complications after induced and spontaneous abortions in Madhya Pradesh, India. Int J Gynecol Obstet 118: S113-20. [Crossref]

54. Henshaw SK, Adewole I, Singh S, Bankole A, Oye-Adeniran B, et al. (2008) Severity and cost of unsafe abortion complications treated in Nigerian hospitals. Int Fam Plan Perspect 34: 40-50. [Crossref]

55. Warren JT, Harvey SM, Henderson JT (2010) Do Depression and Low Self-Esteem Follow Abortion Among Adolescents? Evidence from a National Study. Perspect Sex Reprod Health 42: 230-235. [Crossref]

56. Newitz A. What happens to women denied abortions? This is the first scientific study to find out. [Internet]. io9. 2012 [cited 2016 Mar 15]. Available from: http://io9. com/5958187/what-happens-to-women-denied-abortions-this-is-the-first-scientificstudy-to-find-out

57. DeJong J, Jawad R, Mortagy I, Shepard B (2005) The sexual and reproductive health of young people in the Arab countries and Iran. Reprod Health Matters 13: 49-59. [Crossref]

58. Harries J, Stinson K, Orner P (2009) Health care providers' attitudes towards termination of pregnancy: A qualitative study in South Africa. BMC Public Health 9: 296. [Crossref]

59. Norris A, Bessett D, Steinberg JR, Kavanaugh ML, De Zordo S, et al. (2011) Abortion stigma: a reconceptualization of constituents, causes, and consequences. Women Health Issues 21: S49-54. [Crossref]

60. Norris A, Bessett D, Steinberg JR, Kavanaugh ML, De Zordo S, et al. (2011) Abortion stigma: a reconceptualization of constituents, causes, and consequences. Womens Health Issues 21: S49-54. [Crossref]

61. Barot S (2011) Unsafe abortion: the missing link in global efforts to improve maternal health. Guttmacher Policy Rev 14: 24-28.

62. Cockrill K, Nack A (2013) "I'm Not That Type of Person": Managing the Stigma of Having an Abortion. Deviant Behav 34: 973-990.

Copyright: $(02016$ Ostrach B. This is an open-access article distributed under the terms of the Creative Commons Attribution License, which permits unrestricted use, distribution, and reproduction in any medium, provided the original author and source are credited. 